\title{
Energy flow in jerk-decoupled translatory feed axes
}

B Denkena, H-C Möhring, and P Hesse*

Institute of Production Engineering and Machine Tools, Hanover University, Hanover, Germany

The manuscript was received on 25 July 2006 and was accepted after revision for publication on 5 September 2006.

DOI: 10.1243/0954406JMES455

\begin{abstract}
This paper describes the energy flow in jerk-decoupled translatory feed axes. After an introductory survey on jerk-decoupled feed axes, a system simulation is presented. This system simulation includes a model of the mechanical parts, a model of a synchronous linear motor, and a model of the control circuits. Different energy flows can be simulated using an energy observer. The results are shown in the last section of this paper. With these results, an energy-optimal design of jerk-decoupled linear feed axes can be accomplished.
\end{abstract}

Keywords: feed axis, machine tool, jerk decoupling, energy flow

\section{INTRODUCTION}

The capability, accuracy, and performance of machine tools and production facilities are highly affected by controls and drives. The steady demand for higher dynamics and increased positioning accuracy goes along with the need for high operational availability and high reliability. New approaches to the interaction between the drives and the machine system are necessary.

In order to reach the goal of higher accuracy and higher dynamics, the new technology of jerk decoupling of feed axes was investigated. This technology can be used in rotatory and translatory axes. This paper focuses on translatory axes, but all results are applicable to rotatory drives, too.

In the following, some background information on the need of higher dynamic feed drives and an introduction into jerk-decoupled axes are given. After this section, a system simulation is presented, which includes a model of the mechanical parts, the synchronous linear motor, and the control circuits. With the help of an energy observer, the different energy flows can be visualized. The results are shown in the last section and can be used for an energy-optimal design of jerk-decoupled feed axes.

\footnotetext{
* Corresponding author: Institute of Production Engineering and Machine Tools, Hanover University, Schonebecker Allee, Garbsen 30823, Germany.email: hesse@ifw.uni-hannover.de
}

\section{BACKGROUND}

The goals of higher dynamics and higher absolute positioning accuracy are oppositional. This means that enhancing the dynamics of a system in most cases results in a lower positioning accuracy. Two different types of machine classes are discussed by Heisel et al. [1], high speed machine tools for laser shaping and machines for milling operations. The axes for laser shaping machines feature high accelerations and high velocities in order to realize minor stays in edges of the lasered structure. This is important for obtuse and acute angles in the geometry. On the contrary, the axes in milling machines need to reach high accelerations rather than a high maximum velocity. Instead, the drive forces are much higher compared to laser shaping machines.

Tönshoff et al. [2] describe the influence of the maximum jerk additionally to the acceleration influence. For example, in circular milling processes two longitudinal feed axes have phase-shifted sinusoidal setpoints to generate a circle. In Fig. 1, a diagram is shown, which refers to a circular milling process. On the axis of abscissae, the feed rate and at the axis of ordinate, the radius of the workpiece hole is plotted. On the third axis of the diagram, the maximal acceleration gradient, the jerk, is shown.

On the right-hand side of the borderline, the limiting factor of the dynamics is the acceleration. On the left-hand side, the limit is given through the maximum acceleration gradient - the maximum jerk. 


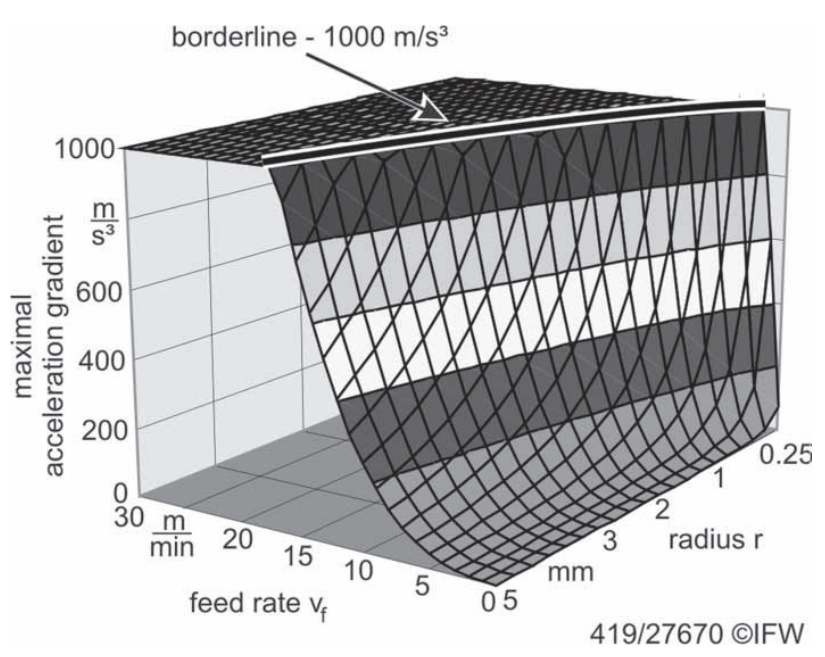

Fig. 1 Maximum jerk of the orthogonal axes during circular driving

This diagram is designed with a borderline of a maximum jerk of $1000 \mathrm{~m} / \mathrm{s}^{3}$. This is actually a very high value for a machine tool. A standard machine available on the market today usually reaches lower maximum values for jerk, so the jerk is indeed an important limiting factor.

\section{JERK DECOUPLING}

In conformity with Newton's third law, a reactive force, such as the active force on the primary part of a linear motor, will act in opposite direction with the same magnitude, from the accelerated mass of the secondary part to the machine and to its foundations [3]. Because a linear motor is able to abruptly raise the force, the machine frame tends to vibrate. These vibrations decrease the accuracy and the workpieces' surface quality will suffer. To decrease these vibrations, a passive filter, i.e. a spring and a damper element, is mounted between the movable secondary part of the linear motor and the frame structure. This configuration is shown in Fig. 2.

This jerk-decoupling principle was patented firstly by the company Krauss-Maffai AG [4] using a linear motor and is also patented by the company Siemens AG [5] using a pinion-shaft or a ball-screw system. Both patents contain the principle of the springdamper system between secondary part and machine frame.

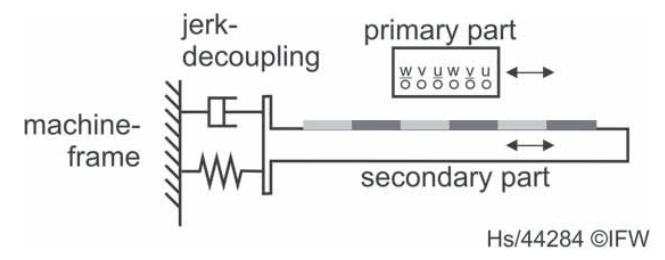

Fig. 2 Principle of jerk-decoupling
Another principle was published by Bubak et al. [3] and is based on mutual relative movement of two machine parts, one of them supporting the workpiece and the other one supporting the tool. This procedure is called the floating principle. The sole relevant parameters for a machining process are the generation of relative movements, relative velocities, and relative accelerations between the workpiece and the tool.

In Germany, the project EffeNDi funded by the Federal Ministry of Education and Research BMBF started to bring the technology of jerk-decoupled axes to the market [6]. For instance, in this project a circular-cycle table unit with a diameter of $500 \mathrm{~mm}$ and a repeat accuracy of $0.01 \mathrm{~mm}$ was realized. A step corresponds to an angle of $30^{\circ}$ and requires a positioning time of $160 \mathrm{~ms}$ without reactive moments to the frame.

\section{MECHANICAL MODEL}

So far, there is no profound knowledge of the energy flow in a jerk-decoupled feed axis, starting from the linear drive and ending at the machine slide. The reason for this incident is that there are no sensors for energy, which can be assembled on the mechanical parts. To solve this problem and to get a clearer idea of the energy flow, a model of the mechanical parts and the drive including the control has been developed at the IFW.

Starting with the mechanical parts, a drawing is shown in Fig. 3. In order to get a better idea of the system, two paths are involved in the mechanical model: the primary path and the secondary path, containing the equivalent frame and the jerkdecoupling slide. The slide of the primary part $m_{\mathrm{P}}$ and the slide of the secondary part $m_{\mathrm{JDC}}$ are guided with longitudinal ball rail systems. The behaviour of the mechanical system is extremely dependent on the forces resulting from the friction in these guides. These friction forces can be broken up into three parts. The first one is lead back to Coulomb [7] and describes the non-velocity-dependent part

$$
f_{\text {fric_c }}(|\nu|)=f_{\mathrm{c}}
$$

The second part is lead back to Reynolds [8] and describes the velocity-dependent part

$$
f_{\text {fric_v }}(|\nu|)=f_{\mathrm{v}} \cdot|\nu|
$$

The last part is lead back to Stribeck [9]. This part describes the dropping of friction with higher velocities in low velocity-ranges

$$
f_{\text {fric_s }}(|\nu|)=f_{\mathrm{h}} \cdot e^{-\beta \cdot|\nu|}
$$




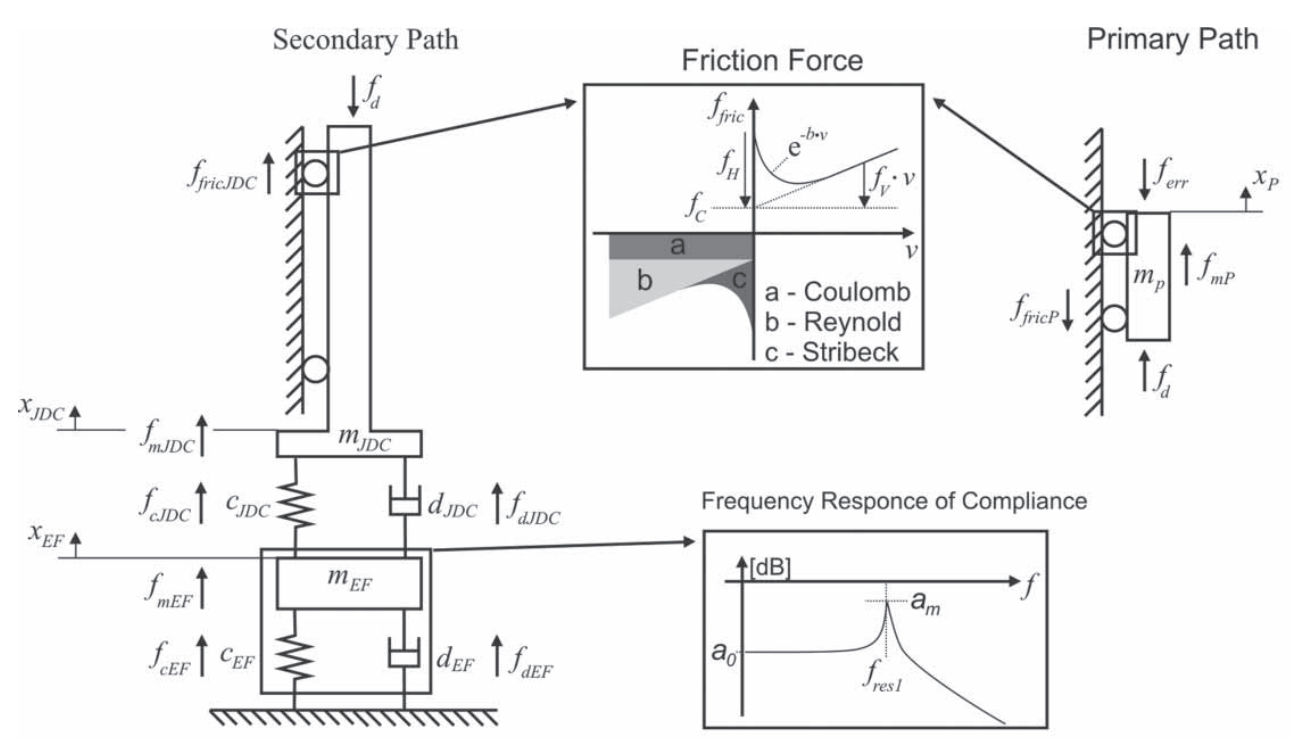

Hs/44249 @IFW

Fig. 3 Mechanical model of a jerk-decoupled axes

All three parts are modelled for the primary and the jerk-decoupled slides, parameterized with measured facts and used in the system simulation.

For some considerations, e.g. within frequency responses, the mechanical model of the friction force has to be simplified. Lapp [10] demonstrated that a single unstressed guiding wagon has these three kinds of frictions as described above. When stressing a guiding wagon, like in this case with the high attractive force of the linear motor, the second part of friction, the Reynolds part, dominates. In the majority of cases from guiding wagons, the Reynolds part at nominal velocity of the linear motor is at minimum 80 per cent of all friction parts. Therefore, while using transfer functions, the friction is limited to the Reynolds part, dependent on the velocity

$$
f_{\text {fric }}(|\nu|)=f_{\mathrm{v}} \cdot|\nu|=b \cdot|\nu|
$$

The newly calculated value $b_{\mathrm{P}}$ for the friction in the primary and $b_{\text {IDC }}$ in the jerk-decoupled slides will be launched. Figure 3 shows three bodies that can be described with the help of force equations. At the primary path, the following equation is valid

$$
-f_{\mathrm{d}}+f_{\text {fric }}-f_{\mathrm{mP}}=0
$$

After a transformation into the frequency domain, the following transfer function is valid for the primary part results

$$
g_{\mathrm{P}}(s)=\frac{x_{\mathrm{P}}}{f_{\mathrm{d}}}=\frac{1}{m_{\mathrm{P}} \cdot s^{2}+b_{\mathrm{P}} \cdot s}
$$

The secondary path comprises two bodies. The equivalent frame embodies the machine structure. This is a PT2-element, the fundamental mode can represent its characteristics. The following equation can be set up for the equivalent frame

$$
-f_{\mathrm{cEF}}-f_{\mathrm{dEF}}-f_{\mathrm{mEF}}-f_{\mathrm{cJDC}}-f_{\mathrm{dJDC}}=0
$$

In reference [11], a measurement strategy is shown that is used to get the parameters for the mass $m_{\mathrm{EF}}$ and those for the spring $c_{\mathrm{EF}}$ of the equivalent frame. This hypothetical factor is extended to take the damping of the equivalent frame $d_{\mathrm{EF}}$ into account. Using the static deformation analysis, the parameter $a_{0}$ can be measured. The parameters $f_{\text {res1 }}$ and $a_{\mathrm{m}}$ can be identified with the help of a modal analysis.

The form of the control parameters is given as

$$
\begin{aligned}
& 10^{\left(\left.a_{0}\right|_{\mathrm{dB}} / 20\right)}=k_{\mathrm{p}, \mathrm{EF}} \\
& 10^{\left(a_{0} \mid \mathrm{dB} / 20\right)}=\frac{k_{\mathrm{p}, \mathrm{EF}}}{\sqrt{4 \cdot D_{\mathrm{EF}}^{4}+\left(2 \cdot D_{\mathrm{EF}} \cdot \sqrt{1-2 \cdot D_{\mathrm{EF}}}\right)^{2}}} \\
& \omega_{\mathrm{m} 1}=2 \cdot \pi \cdot f_{\mathrm{res} 1}=\omega_{\mathrm{O}, \mathrm{EF}} \cdot \sqrt{1-2 \cdot D_{\mathrm{EF}}^{2}}
\end{aligned}
$$

For the use of the equivalent element, the mechanical parameters can be calculated as

$$
\begin{aligned}
& m_{\mathrm{EF}}=\frac{1}{k_{\mathrm{p}, \mathrm{EF}} \cdot \omega_{0, \mathrm{EF}}^{2}} \\
& d_{\mathrm{EF}}=\frac{2 \cdot D_{\mathrm{EF}}}{k, E F} \cdot \omega_{0, \mathrm{EF}} \\
& c_{\mathrm{EF}}=\frac{1}{k_{\mathrm{p}, \mathrm{EF}}}
\end{aligned}
$$


The second body of the secondary path is described by the following equation

$$
-f_{\mathrm{cJDC}}-f_{\mathrm{dJDC}}-f_{\mathrm{mJDC}}-f_{\mathrm{d}}-f_{\text {fricJDC }}=0
$$

The force $f_{\text {fricjDC }}$ has the same structure as the force $f_{\text {fricP }}$ in the primary path, but different parameter values, because different guides are used. The force $f_{\mathrm{d}}$ is the same force as in equation (5). This means that the linear motor generates two forces of the same magnitude. The first one lies in the primary path and the second one in the secondary path.

To get the transfer function of the equivalent frame and the jerk-decoupling slide, it is necessary to combine equations (7) and (14). After transforming into the frequency domain, the following transfer function of the equivalent frame is concluded

$$
\begin{aligned}
& g_{\mathrm{EF}}(s)=\frac{x_{\mathrm{EF}}}{f_{\mathrm{d}}}=-\left(d_{\mathrm{JDC}} \cdot s+c_{\mathrm{JDC}}\right) /\left[\left(m_{\mathrm{EF}} \cdot m_{\mathrm{JDC}}\right) \cdot s^{4}\right. \\
& +\left(\begin{array}{l}
m_{\mathrm{EF}} \cdot d_{\mathrm{JDC}}+m_{\mathrm{EF}} \cdot b_{\mathrm{JDC}} \\
+d_{\mathrm{EF}} \cdot m_{\mathrm{JDC}}+d_{\mathrm{JDC}} \cdot m_{\mathrm{JDC}}
\end{array}\right) \cdot s^{3} \\
& +\left(\begin{array}{l}
m_{\mathrm{EF}} \cdot c_{\mathrm{JDC}}+d_{\mathrm{EF}} \cdot d_{\mathrm{JDC}} \\
+d_{\mathrm{EF}} \cdot b_{\mathrm{JDC}}+d_{\mathrm{JDC}} \cdot b_{\mathrm{JDC}} \\
+c_{\mathrm{EF}} \cdot m_{\mathrm{JDC}}+c_{\mathrm{JDC}} \cdot m_{\mathrm{JDC}}
\end{array}\right) \cdot s^{2} \\
& +\left(\begin{array}{l}
d_{\mathrm{EF}} \cdot c_{\mathrm{JDC}}+c_{\mathrm{EF}} \cdot d_{\mathrm{JDC}} \\
+c_{\mathrm{EF}} \cdot b_{\mathrm{JDC}}+c_{\mathrm{JDC}} \cdot b_{\mathrm{JDC}}
\end{array}\right) \cdot s \\
& +c_{\mathrm{EF}} \cdot c_{\mathrm{JDC}}
\end{aligned}
$$

The transfer function of the jerk-decoupling slide can be calculated the same way

$$
\begin{aligned}
& g_{\mathrm{JDC}}(s)=\frac{x_{\mathrm{JDC}}}{f_{\mathrm{d}}}= \\
& -\left[m_{\mathrm{EF}} \cdot s^{2}+\left(d_{\mathrm{EF}}+d_{\mathrm{JDC}}\right) \cdot s+\left(c_{\mathrm{EF}}+c_{\mathrm{JDC}}\right)\right] / \\
& \quad\left[\begin{array}{c}
\left(m_{\mathrm{EF}} \cdot m_{\mathrm{JDC}}\right) \cdot s^{4} \\
+\left(\begin{array}{c}
m_{\mathrm{EF}} \cdot d_{\mathrm{JDC}}+m_{\mathrm{EF}} \cdot b_{\mathrm{JDC}} \\
+d_{\mathrm{EF}} \cdot m_{\mathrm{JDC}}+d_{\mathrm{JDC}} \cdot m_{\mathrm{JDC}}
\end{array}\right) \cdot s^{3} \\
+\left(\begin{array}{c}
m_{\mathrm{EF}} \cdot c_{\mathrm{JDC}}+d_{\mathrm{EF}} \cdot d_{\mathrm{JDC}} \\
+d_{\mathrm{EF}} \cdot b_{\mathrm{JDC}}+d_{\mathrm{JDC}} \cdot b_{\mathrm{JDC}} \\
+c_{\mathrm{EF}} \cdot m_{\mathrm{JDC}}+c_{\mathrm{JDC}} \cdot m_{\mathrm{JDC}}
\end{array}\right) \cdot s^{2} \\
+\left(\begin{array}{c}
d_{\mathrm{EF}} \cdot c_{\mathrm{JDC}}+c_{\mathrm{EF}} \cdot d_{\mathrm{JDC}} \\
+c_{\mathrm{EF}} \cdot b_{\mathrm{JDC}}+c_{\mathrm{JDC}} \cdot b_{\mathrm{JDC}}
\end{array}\right) \cdot s \\
+c_{\mathrm{EF}} \cdot c_{\mathrm{JDC}}
\end{array}\right]
\end{aligned}
$$

Figure 3 shows the linear motor beeing placed between the primary path and the jerk-decoupling slide of the secondary path. In order to get the transfer function of the linear motor, which is extremely important for designing the velocity-control circuit, equations (6) and (16) are merged together

$$
\begin{aligned}
& g_{\mathrm{P}-\mathrm{JDC}}(s)=\frac{x_{\mathrm{EF}}-x_{\mathrm{IDC}}}{f_{\mathrm{d}}} \\
& =-\left[\left(m_{\mathrm{EF}} \cdot m_{\mathrm{JDC}}+m_{\mathrm{EF}} \cdot m_{\mathrm{P}}\right) \cdot s^{4}\right. \\
& +\left(\begin{array}{l}
m_{\mathrm{EF}} \cdot d_{\mathrm{JDC}}+m_{\mathrm{EF}} \cdot b_{\mathrm{JDC}} \\
+d_{\mathrm{EF}} \cdot m_{\mathrm{JDC}}+d_{\mathrm{JDC}} \cdot m_{\mathrm{JDC}} \\
+m_{\mathrm{EF}} \cdot b_{\mathrm{P}}+d_{\mathrm{EF}} \cdot m_{\mathrm{P}}+d_{\mathrm{JDC}} \cdot m_{\mathrm{P}}
\end{array}\right) \cdot s^{3} \\
& +\left(\begin{array}{l}
m_{\mathrm{EF}} \cdot c_{\mathrm{JDC}}+d_{\mathrm{EF}} \cdot d_{\mathrm{JDC}} \\
+d_{\mathrm{EF}} \cdot b_{\mathrm{JDC}}+d_{\mathrm{JDC}} \cdot b_{\mathrm{JDC}} \\
+c_{\mathrm{EF}} \cdot m_{\mathrm{JDC}}+c_{\mathrm{JDC}} \cdot m_{\mathrm{JDC}} \\
+d_{\mathrm{EF}} \cdot b_{\mathrm{P}}+d_{\mathrm{JDC}} \cdot b_{\mathrm{P}} \\
+c_{\mathrm{EF}} \cdot c_{\mathrm{JDC}} \cdot m_{\mathrm{P}}
\end{array}\right) \cdot s^{2} \\
& \left.+\left(\begin{array}{c}
d_{\mathrm{EF}} \cdot c_{\mathrm{JDC}}+c_{\mathrm{EF}} \cdot d_{\mathrm{JDC}} \\
+c_{\mathrm{EF}} \cdot b_{\mathrm{JDC}}+c_{\mathrm{JDC}} \cdot b_{\mathrm{JDC}} \\
+c_{\mathrm{EF}} \cdot c_{\mathrm{JDC}} \cdot b_{\mathrm{P}}
\end{array}\right) \cdot s+c_{E F} \cdot c_{\mathrm{JDC}}\right] / \\
& {\left[s \cdot\left(m_{\mathrm{P}} \cdot s+b_{\mathrm{P}}\right)\right.} \\
& \left.\left(\begin{array}{c}
m_{\mathrm{EF}} \cdot m_{\mathrm{JDC}} \cdot s^{4} \\
+\left(\begin{array}{c}
m_{\mathrm{EF}} \cdot d_{\mathrm{JCC}}+m_{\mathrm{EF}} \cdot b_{\mathrm{JDC}} \\
+d_{\mathrm{EF}} \cdot m_{\mathrm{JDC}}+d_{\mathrm{JDC}} \cdot m_{\mathrm{JDC}}
\end{array}\right) \cdot s^{3} \\
+\left(\begin{array}{c}
m_{\mathrm{EF}} \cdot c_{\mathrm{JDC}}+d_{\mathrm{EF}} \cdot d_{\mathrm{JDC}} \\
+d_{\mathrm{EF}} \cdot b_{\mathrm{JDC}}+d_{\mathrm{JDC}} \cdot b_{\mathrm{JDC}} \\
+c_{\mathrm{EF}} \cdot m_{\mathrm{JDC}}+c_{\mathrm{JDC}} \cdot m_{\mathrm{JDC}}
\end{array}\right) \cdot s^{2} \\
+\left(\begin{array}{c}
d_{\mathrm{EF}} \cdot c_{\mathrm{JDC}}+c_{\mathrm{EF}} \cdot d_{\mathrm{JDC}} \\
+c_{\mathrm{EF}} \cdot b_{\mathrm{JDC}}+c_{\mathrm{JDC}} \cdot b_{\mathrm{JDC}}
\end{array}\right) \cdot s \\
+c_{\mathrm{EF}} \cdot c_{\mathrm{JDC}}
\end{array}\right)\right]
\end{aligned}
$$

These transfer functions describe the complete mechanical model comprising the primary part, the jerk-decoupling slide, the equivalent frame, and the linear motor. In reference [10], Lapp used the same mechanical model for the primary part. The difference between the system of Lapp and the system examined in this paper is the application of a jerkdecoupling technology. A validation of the primary path and the friction in this path are done in reference [10] and show good results. A validation for the secondary path cannot be demonstrated yet at this point, because the jerk-decoupling technology is very new and the prototype is currently under construction. The system-simulation described in the next chapter is meant to get a general idea of the performance before building up a prototype. 
For this reason, a validation of the secondary part cannot be shown at this stage of the project.

\section{SYSTEM SIMULATION}

In this section, the mechanical model will be integrated into a system simulation. Therefore, a model of the linear motor and the control circuits has been developed. In most cases, the linear motors in machines are based on the synchronous principle. In order to get the best approximation of the linear synchronous motor, a model of a rotating-field main machine is applied. Three different ways of controls are commonly used, the trapezoidal commutation, the sinusoidal commutation, and the field oriented control. Field oriented control provides a smooth motion at slow speeds as well as an efficient operation at high speeds [12]. For this reason, the field oriented control is used to control linear motors in machines, especially in machine tools.

A model of a field-oriented synchronous linear motor consists of three parts. The first part is the transformation between the stator and the rotor coordinate system, the second is the motor model itself and the third part is the transformation between the rotor and the stator coordinate system.

Beginning with the transformation from the stator orthogonal coordinate system to the rotor coordinate system, the voltages $u_{u}, u_{v}$, and $u_{w}$ can be transformed to the voltages $u_{d}$ and $u_{q}$ with the help of the rotor angle $\theta_{r}$

$$
\begin{aligned}
& {\left[\begin{array}{l}
u_{\alpha} \\
u_{\beta}
\end{array}\right]=\frac{2}{3} \cdot\left[\begin{array}{ccc}
1 & -\frac{1}{2} & -\frac{1}{2} \\
0 & \frac{\sqrt{3}}{2} & -\frac{\sqrt{3}}{2}
\end{array}\right] \cdot\left[\begin{array}{l}
u_{u} \\
u_{v} \\
u_{w}
\end{array}\right]} \\
& {\left[\begin{array}{l}
u_{d} \\
u_{q}
\end{array}\right]=\left[\begin{array}{ll}
\cos \left(\theta_{\mathrm{r}}\right) & \sin \left(\theta_{\mathrm{r}}\right) \\
-\sin \left(\theta_{\mathrm{r}}\right) & \cos \left(\theta_{\mathrm{r}}\right)
\end{array}\right] \cdot\left[\begin{array}{l}
u_{\alpha} \\
u_{\beta}
\end{array}\right]}
\end{aligned}
$$

After transforming the voltages into the rotor coordinate system, a model of the linear motor itself is required. Olrik [13] developed such a model. A scheme is shown in Fig. 4.

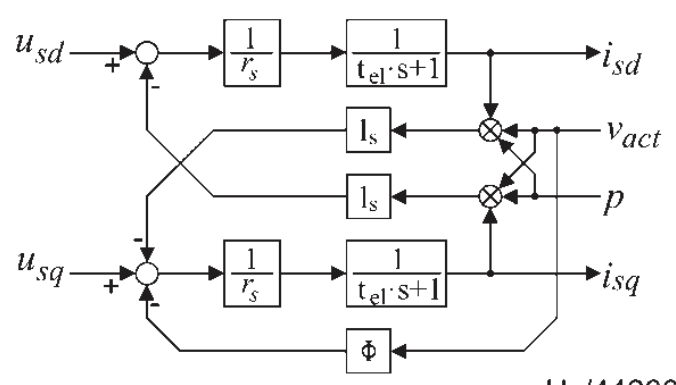

$\mathrm{H} \$ / 44266$ 이 FW

Fig. 4 Model of a synchronous machine
Different motor parameters are involved in it, e.g. the resistance $r_{\mathrm{s}}$, the inductivity $l_{\mathrm{s}}$, and the electric delay time constant $t_{\mathrm{el}}$

$$
t_{\mathrm{el}}=\frac{l_{\mathrm{s}}}{r_{\mathrm{s}}}
$$

The variable $p$ specifies the number of pairs of poles in the stator and the variable $v_{\text {act }}$ describes the actual velocity of the machine. When using a typical linear motor with one pair of poles, the equation for the motor model is given as

$$
\begin{aligned}
\frac{\mathrm{d}}{\mathrm{d} t}\left[\begin{array}{l}
i_{d} \\
i_{q}
\end{array}\right]=\left[\begin{array}{cc}
-\frac{r_{\mathrm{s}}}{l_{\mathrm{s}}} & v_{\mathrm{act}} \\
-\frac{r_{\mathrm{s}}}{l_{\mathrm{s}}} & -v_{\text {act }}
\end{array}\right] \cdot\left[\begin{array}{c}
i_{d} \\
i_{q}
\end{array}\right]+\left[\begin{array}{cc}
\frac{1}{l_{\mathrm{s}}} & 0 \\
\frac{1}{l_{\mathrm{s}}} & 0
\end{array}\right] \\
\cdot\left[\begin{array}{l}
u_{d} \\
u_{q}
\end{array}\right]-\left[\begin{array}{c}
0 \\
\Phi \cdot v_{\text {act }}
\end{array}\right] \\
f_{\mathrm{d}}=\frac{3}{2} \cdot \Phi \cdot i_{q}
\end{aligned}
$$

In equation (21) and in Fig. 4, the cross coupling of the currents $i_{d}$ and $i_{q}$ is visible. After calculating these currents and the drive force $f_{\mathrm{d}}$, another transformation from the rotor coordinate system to the orthogonal coordinate system is needed

$$
\begin{aligned}
& {\left[\begin{array}{l}
i_{\alpha} \\
i_{\beta}
\end{array}\right]=\left[\begin{array}{cc}
\cos \left(\theta_{\mathrm{r}}\right) & -\sin \left(\theta_{\mathrm{r}}\right) \\
\sin \left(\theta_{\mathrm{r}}\right) & \cos \left(\theta_{\mathrm{r}}\right)
\end{array}\right] \cdot\left[\begin{array}{l}
i_{d} \\
i_{q}
\end{array}\right]} \\
& {\left[\begin{array}{c}
i_{u} \\
i_{v} \\
i_{w}
\end{array}\right]=\left[\begin{array}{cc}
1 & 0 \\
0 & \frac{\cos (\pi / 6)}{2} \\
0 & -\frac{\cos (\pi / 6)}{2}
\end{array}\right] \cdot\left[\begin{array}{c}
i_{\alpha} \\
i_{\beta}
\end{array}\right]}
\end{aligned}
$$

In front of the model of the synchronous machine, the control circuits are placed. A standard cascade control with a current, velocity, and position control is used. This part, the modelling of the control, is state of the art [10] and therefore not described within this paper.

In order to calculate the control parameters in the current control circuit, the field-oriented control algorithm is applied. Usually, the currents of the three phases are visualized in a polar coordinate system, but for a linear motor this is suboptimal. A new method to visualize a field-oriented control is shown in Fig. 5. On the axis of abscissa, the position on the primary part is shown. The term $2 \cdot \tau_{\mathrm{g}}$ represents the doubled value of the pole's width, so it is the active length of the primary part. Every $2 / 3 \cdot \tau_{\mathrm{g}}$, a coil is placed for the three phase system and a vector for the amplitude of each voltage is calculated. 


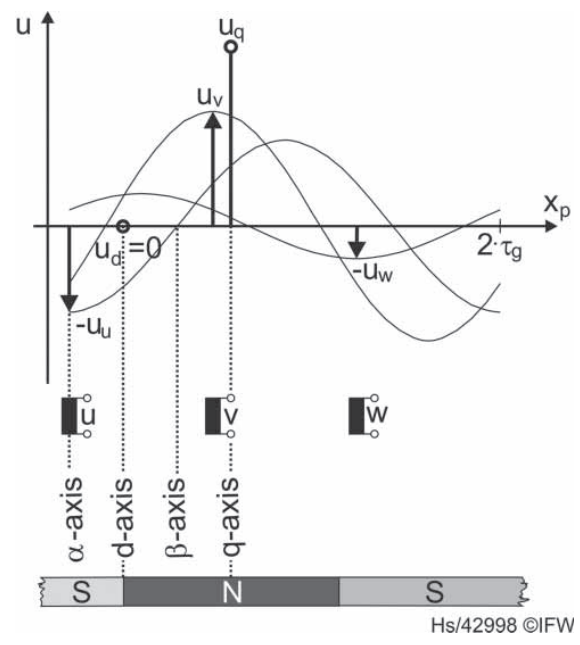

Fig. 5 Coordinate demonstration for linear motors

Each vector represents the amplitude of a sinus wave and the associated phase shift. Having the secondary path movable underneath the axis of abscissa, the $d$ - and $q$-axes can be drawn into the diagram. After the summation of all amplitudes of the three phases, the voltages $u_{d}$ and $u_{q}$ can be calculated. Figure 5 shows a good working field orientation meaning the voltage $u_{d}$ is controlled to zero and the voltage $u_{q}$ to the current set point.

\section{ENERGY OBSERVER}

The previous chapter describes the simulation environment with the primary and secondary path of the jerk-decoupled axes with the use of a model of a synchronous linear direct drive. Based on this simulation an energy observer has been developed.

The physical base of the differential work is defined in reference [14]. The primary path contains three kinds of energy, the potential if using the axis in the vertical manner, the kinetic, and the friction energy. The potential differential work $\mathrm{d} w_{\mathrm{P}, \text { pot }}$ and the potential work $\Delta e_{\mathrm{P} \text {,pot }}$ are given as

$$
\begin{aligned}
& \mathrm{d} w_{\mathrm{P}, \text { pot }}=m_{\mathrm{P}} \cdot g \cdot v_{\mathrm{P}} \cdot v / \mathrm{h} \\
& \Delta e_{\mathrm{P}, \mathrm{pot}}^{x_{\mathrm{P}}=0}=m_{\mathrm{P}} \cdot g \cdot x_{\mathrm{P}} \cdot v / \mathrm{h}
\end{aligned}
$$

The parameter $v / h$ can be set to one, if the axis is used vertically and is set to zero, if the axis is used horizontally. The same equations can be set up for the kinetic energy

$$
\begin{aligned}
& \mathrm{d} w_{\mathrm{P}, \mathrm{kin}}=m_{\mathrm{P}} \cdot a_{\mathrm{P}} \cdot v_{\mathrm{P}} \\
& \Delta e_{\mathrm{P}, \mathrm{kin}}^{\nu_{\mathrm{P}}=0}=\frac{1}{2} \cdot m_{\mathrm{P}} \cdot v_{\mathrm{P}}^{2}
\end{aligned}
$$

and the friction energy

$$
\begin{aligned}
& \mathrm{d} w_{\mathrm{P}, \text { fric }}=f_{\text {fric }, \mathrm{P}} \cdot v_{\mathrm{P}} \\
& \Delta e_{\mathrm{P}, \text { fric }}=\int \mathrm{d} w_{\mathrm{P}, \text { fric }} \mathrm{d} t
\end{aligned}
$$

The differential work of the friction is calculated as the product of the friction force $f_{\text {fric,P }}$ and the actual velocity. To obtain the work, the differential work is to be integrated over the time. Symbolically, this integration represents a thermal energy storage, in which the friction's differential work will be transferred. With this understanding, a leakage sink of the friction energy can be shown.

Analogous to the primary path the equations can be set up for the equivalent frame and the jerkdecoupling slide. Starting with the potential work

$$
\begin{aligned}
& \mathrm{d} w_{\mathrm{EF}, \mathrm{pot}}=m_{\mathrm{EF}} \cdot g \cdot v_{\mathrm{EF}} \cdot v / \mathrm{h} \\
& \Delta e_{\mathrm{EF}, \text { pot }}^{x_{\mathrm{P}}}=m_{\mathrm{EF}} \cdot g \cdot x_{\mathrm{EF}} \cdot v / \mathrm{h}
\end{aligned}
$$

and continuing with the kinetic work

$$
\begin{aligned}
& \mathrm{d} w_{\mathrm{EF}, \mathrm{kin}}=m_{\mathrm{EF}} \cdot a_{\mathrm{EF}} \cdot v_{\mathrm{EF}} \\
& \Delta e_{\mathrm{EF}, \mathrm{kin}}^{\nu_{\mathrm{EF}}=0}=\frac{1}{2} \cdot m_{\mathrm{EF}} \cdot v_{\mathrm{EF}}^{2}
\end{aligned}
$$

On the one hand, the equivalent frame comprises no friction; on the other hand there is material damping. The work in this material damping can be calculated as follows

$$
\begin{aligned}
& \mathrm{d} w_{\mathrm{EF}, \mathrm{damp}}=d_{\mathrm{EF}} \cdot v_{\mathrm{EF}}^{2} \\
& \Delta e_{\mathrm{EF}, \mathrm{damp}}^{\nu_{\mathrm{EF}}=0}=\int \mathrm{d} w_{\mathrm{EF}, \mathrm{damp}} \mathrm{d} t
\end{aligned}
$$

Considering the energy, it is important to have the same energy storage as the friction energy. With the help of this view, a leakage sink of the damping energy can also be shown.

The work stored within the spring is given as

$$
\begin{aligned}
& \mathrm{d} w_{\mathrm{EF}, \text { elast }}=c_{\mathrm{EF}} \cdot x_{\mathrm{EF}} \cdot v_{\mathrm{EF}} \\
& \Delta e_{\mathrm{EF}, \text { elast }}^{x_{\mathrm{EF}}=0}=\frac{1}{2} \cdot c_{\mathrm{EF}} \cdot x_{\mathrm{EF}}^{2}
\end{aligned}
$$

Analogous to the equivalent frame, the energy equations for the jerk-decoupling slide can be calculated, starting with the potential work

$$
\begin{aligned}
& \mathrm{d} w_{\mathrm{JDC}, \mathrm{pot}}=m_{\mathrm{JDC}} \cdot g \cdot v_{\mathrm{JDC}} \cdot v / \mathrm{h} \\
& \Delta e_{\mathrm{JDC}, \mathrm{pot}}^{x_{\mathrm{JC}}=0}=m_{\mathrm{JDC}} \cdot g \cdot x_{\mathrm{JDC}} \cdot v / \mathbf{h}
\end{aligned}
$$


the kinematic work

$$
\begin{aligned}
& \mathrm{d} w_{\mathrm{JDC}, \mathrm{kin}}=m_{\mathrm{JDC}} \cdot a_{\mathrm{JDC}} \cdot v_{\mathrm{JDC}} \\
& \Delta e_{\mathrm{JDC}, \mathrm{kin}}^{\nu_{\mathrm{JDC}}=0}=\frac{1}{2} \cdot m_{\mathrm{JDC}} \cdot v_{\mathrm{JDC}}^{2}
\end{aligned}
$$

the elastic work

$$
\begin{aligned}
& \mathrm{d} w_{\mathrm{JDC}, \text { elast }}=c_{\mathrm{JDC}} \cdot\left(x_{\mathrm{JDC}}-x_{\mathrm{EF}}\right) \cdot\left(\nu_{\mathrm{JDC}}-v_{\mathrm{EF}}\right) \\
& \Delta e_{\mathrm{JDC}, \text { elast }}^{\left(x_{\mathrm{IJC}}-x_{\mathrm{FF}}\right)=0}=\frac{1}{2} \cdot c_{\mathrm{JDC}} \cdot\left(x_{\mathrm{JDC}}-x_{\mathrm{EF}}\right)^{2}
\end{aligned}
$$

the work caused by damping

$$
\begin{aligned}
& \mathrm{d} w_{\mathrm{JDC}, \mathrm{damp}}=d_{\mathrm{JDC}} \cdot\left(\nu_{\mathrm{JDC}}-v_{\mathrm{EF}}\right)^{2} \\
& \Delta e_{\mathrm{JDC}, \mathrm{damp}}^{\left(\nu_{\mathrm{IJC}}-\nu_{\mathrm{EF}}\right)=0}=\int \mathrm{d} w_{\mathrm{JDC}, \mathrm{damp}} \mathrm{d} t
\end{aligned}
$$

and the work caused by friction

$$
\begin{aligned}
& \mathrm{d} w_{\mathrm{JDC}, \text { fric }}=f_{\text {fric }, \mathrm{P}} \cdot v_{\mathrm{JDC}} \\
& \Delta e_{\mathrm{JDC}, \text { fric }}^{\nu_{\mathrm{JDC}}=0}=\int \mathrm{dw}_{\mathrm{JDC}, \text { fric }} \mathrm{dt}
\end{aligned}
$$

Using these equations, the complete energy flow within the mechanics can be described. The model of the synchronous linear direct drive is placed ahead of the mechanical model within the system simulation. For calculating the leakage of the drive, the sum-current within the rotating coordinate system has to be determined. With the use of equation (21), the sum-current is shown

$$
i_{\mathrm{s}}=\sqrt{i_{q}^{2}+i_{d}^{2}}
$$

Using the sum-current the differential leakage work and the leakage work of the drive results

$$
\begin{aligned}
\mathrm{d} w_{\mathrm{SM}, \text { leak }} & =r_{\mathrm{s}} \cdot i_{\mathrm{s}}^{2} \\
\Delta e_{\mathrm{SM}, \text { leak }} & =\int \mathrm{d} w_{\mathrm{SM}, \text { leak }} \mathrm{d} t
\end{aligned}
$$

The parameter $r_{\mathrm{s}}$ outlines the internal resistance of the windings. It is easy to conclude that the sum-current is always positive, so the leakage work is always positive as well. This is independent from the drive being used as a motor or a generator. Therefore, the degree of efficiency is always lower than one.

\section{ENERGY-FLOW IN JERK-DECOUPLED AXES}

First, the energy flow in a conventional linear motor without jerk decoupling will be shown. These results are the base for the comparison of the jerkdecoupling principle with a standard application of the linear motor.

The foundation of all following results is the motion profile shown at the top of Fig. 6. It consists of two point-to-point (PTP) movements, the first into the positive position $s_{\mathrm{P}}$ and the second into the negative position $-s_{\mathrm{P}}$. The motion profile, the differential work, and the work within the mechanical parts of the primary path are shown below.

All diagrams refer to a horizontal axis, so there is no potential energy flow shown. The energy flow of the primary path is quite easy to understand, the kinetic energy is shown in the positioning times of the axes and the only leakage is the friction in the guides. In this example, the linear motor has to be designed for a maximum power of $2275 \mathrm{~W}$ in order to faciliate this profile. The amount of the maximum work is pictured in the sum-work at $186 \mathrm{~J}$, but after this point the kinetic energy recovers so that the used work for this profile is $108 \mathrm{~J}$.

Starting from this calculation, the next step is the simulation of the work in the equivalent frame and the jerk-decoupling slide. The first body, the equivalent frame, can be neglected because the movement and the velocities are small and the differential work is tiny. Because of this, the focus is now set on the jerk-decoupling slide.

At the top of Fig. 7, the movement profile of the secondary part is shown. The profile, the work of the primary path, and the jerk-decoupling are shown below.
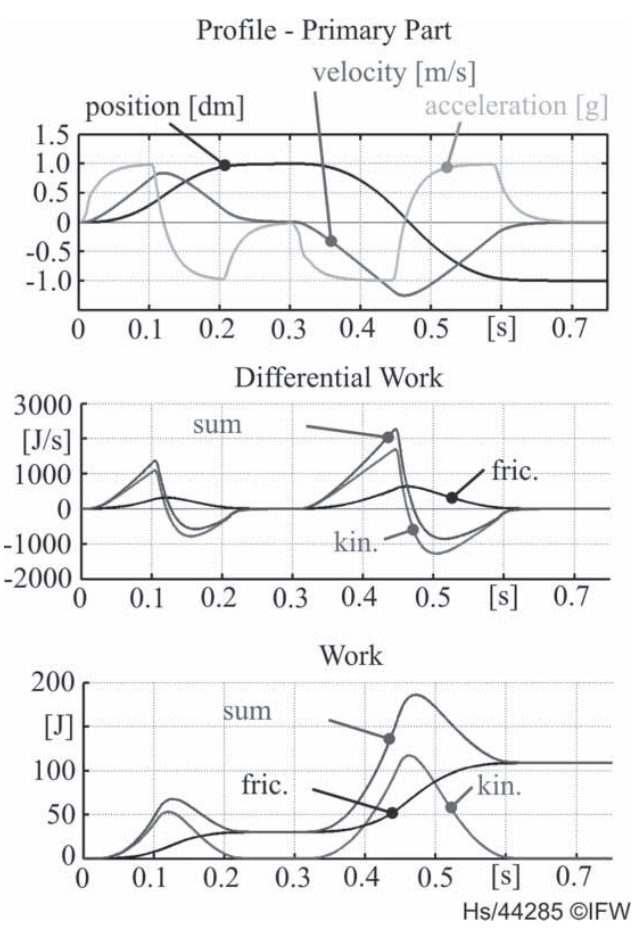

Fig. 6 Differential work and work within the primary path 

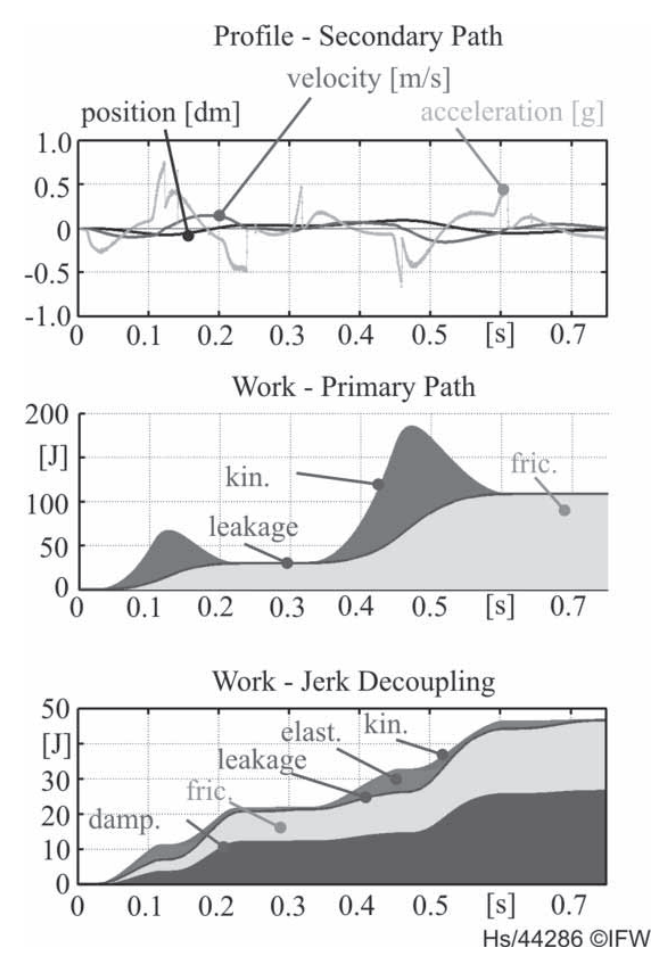

Fig. 7 Work in the jerk-decoupling path

The kinetic and the elastic work is zero after finishing the profile. The leakage of the secondary path consists of a friction and of a damping part.

In this case - on top of the $108 \mathrm{~J}$ needed for the primary path - there is a leakage of $47 \mathrm{~J}$ for the secondary path. Therefore, the efficiency factor of a jerk-decoupled application is always lower compared to a standard linear motor application. Apart from this, the advantages of the jerk decoupling are much higher possible control parameters as well as higher possible dynamics of the system.

In a synchronous linear direct drive, the force is nearly proportional to the current and the velocity is nearly proportional to the voltage. Using a jerkdecoupling, the relative velocity between the movement of the primary and the secondary part is higher than in a conventional axis. The forces remain the same. Therefore, in conclusion, considering a jerk-decoupled application, there is a higher voltage applied to the linear motor at the same current level. As shown in the equations (50) and (51), the leakage of a synchronous linear motor is quadratic to its current. Therefore, the result of using jerk decoupling is a higher voltage, but not a higher leakage in the synchronous machine.

\section{VARIATIONS OF PARAMETERS}

In this chapter, variations of the parameters in the jerk-decoupling path are investigated. At first,

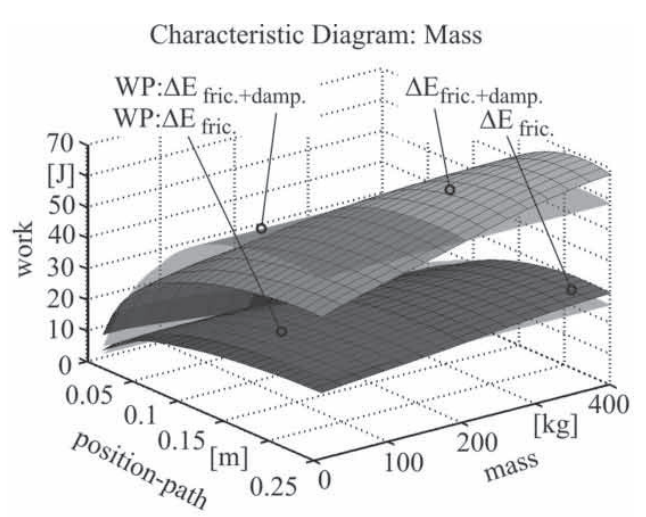

Characteristic Diagram: Spring Stiffness

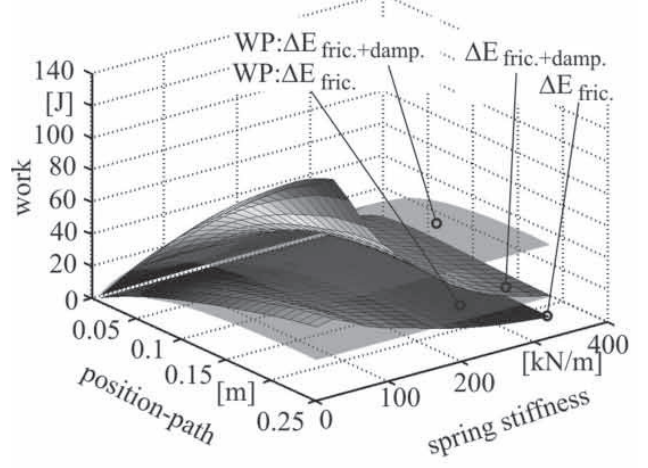

Characteristic Diagram: Attenuation Constant

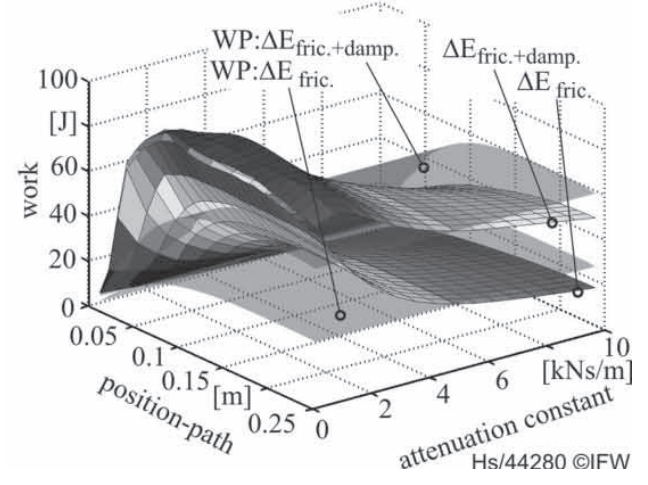

Fig. 8 Variation of the jerk-decoupling parameters

parameters for the path are calculated with the method demonstrated in reference $[\mathbf{1 1}]$.

For this application, a mass of $m_{\mathrm{JDC}}=200 \mathrm{~kg}$, a spring stiffness of $c_{\mathrm{JDC}}=160000 \mathrm{~N} / \mathrm{m}$, and an attenuation constant of $d_{\mathrm{JDC}}=6500 \mathrm{~N} \cdot \mathrm{s} / \mathrm{m}$ are given. In the upper part of Fig. 8, the parameter $m_{\mathrm{JDC}}$, in the middle the parameter $c_{\mathrm{JDC}}$, and lower part the parameter $d_{\mathrm{JDC}}$ are varied. In this characteristic diagram, only the leakage energies are shown depending on the position path and the variable parameters. Two solid faces are drawn; the lower one represents the friction energy and the upper one represents the work of the friction and the damping energy. The working points are drawn with the transparent faces. 
Starting with the variation of the mass, it is easy to see that a lower mass causes a lower leakage work. Generally, in mechanical engineering the light weight construction is considered to be state of the art, but this is done in the consideration of minimizing the kinetic energies in order to increase the maximum acceleration, or to use a smaller motor at the same acceleration. However, in the case at hand the light weight construction of the jerk-decoupling slide is employed to minimize the leakage work.

Having a look on the middle diagram in Fig. 8, the dependence of the leakage work on the spring stiffness is shown. In this case, it is important to have a high stiffness to decrease the leakage work within the friction and the damping.

In Fig. 8, the dependency of the leakage work on the damping constant is shown within the lower diagram. A different view of the friction and the damping energy is necessary here. Considering the friction, it is important to have a high attenuation constant. The damping work has a minimum at small attenuation constants and levels out as they increase. Anyway, the optimum over all is a high attenuation constant.

\section{CONCLUSIONS}

The modelling, analysis, and design of jerkdecoupled axes have been discussed. The main subject contemplated in this paper was the analysis of the energies within the mechanical parts and the synchronous linear direct drive. After the modelling and the design of a system simulation, an energy observer was built.

The results of this paper show that jerk-decoupled axes require more energy than a comparable standard application with a direct drive. The reasons herefore are the friction of the secondary path and the damping of the jerk decoupling. These effects have to be minimized in order to get an efficient and cost-effective high speed axes.

\section{ACKNOWLEDGEMENT}

The authors wish to thank the EC for the possibility to generate this research within the integrated project 'Next Generation Production Systems' (NEXT).

\section{REFERENCES}

1 Heisel, U., Feinauer, A., and Rudlaff, T. Forderungen an Hochgeschwindigkeitsmaschinen. J. wt-Produktion und Management, 85(4), 1995, 155-161.
2 Tönshoff, H. K., Ben Amor, R., Kaak, R. and Urban, B. Fräsen ohne Tempolimit? J. wt-Werkstattstechnik, 1999, 7/8, 365-368.

3 Bubak, A., Soucek, P., and Zeleny, J. New principles for design of highly dynamic machine tools. Proceedings of the International Conference ICPR-17 (Ed M. P. Deisenroth), Blacksburg, Virginia, USA, 3-7 August 2003, (Virginia Polytechnic Institut and State University). ISBN 09721257-3-6.

4 Stoiber, D. Impulsentkoppelter Direktantrieb. German patent, DE 19810996 Al, 16 September 1999.

5 Knorr, M. Impulsgekoppelter Transmissionsantrieb. German patent, DE 10117460 Al, 7 November 2002.

6 Berkemer, J. Conclution presentation EffeNDi, proceedings, Stuttgart, 10 November 2004.

7 Coulomb, C. A. Théorie des machines simples, en ayant égard au frottement de leurs parties, et à la roideur dews cordages. Adaptive control of mechanical manipulators, 1875, pp. 161-342 (Addison-Wesley, Reading, MA).

8 Reynolds, 0 . On the theory of lubrication and its application to M. Beuchamp Tower's experiments, including an experimental determination of the viscosity of olive oil. Phil. Trans. Royal Soc., 1886, 177, 157-234.

9 Stribeck, R. Die wesentlichen Eigenschaften der Gleitund Rollenlager. J. Verein Deutscher Ingenieure, 1902, 6, $1342-1348$.

10 Lapp, C. Indirekte Erfassung und Regelung von Bearbeitungskräften an direkt getriebenen Vorschubeinheiten, 2002 (Fortschritt-Berichte VDI, Düsseldorf).

11 Neugebauer, R., Riedel, M., and Drossel, W.-G. Modellbasierte Auslegung von Koppelelementen. J. antriebstechnik, 2004, 11, 70-74.

12 Copley Controls Corp. Available from www.copleycontrols.com/motion/downloads/pdf/field-orientedcontrol.pdf, 2003.

13 Orlik, B. Zum Betriebsverhalten mikrorechnergeregelter und pulswechselrichtergespeister Synchronmaschinen mit Permanentmagneterregung. Doctoral Thesis of the Fakultät für Maschinenbau und Elektrotechnik der Technischen Universität Carolo-Wilhelmina zu Braunschweig, 1987.

14 Beitz, W. and Grote, K.-H. Handbook of mechanical engineering, (Springer-Verlag, Berlin, Heidelberg, New York) (ISBN 3-540-62467-8, 1997).

\section{APPENDIX}

\section{Notation}

$a_{0} \quad$ compliance in static case

$a_{\mathrm{m}} \quad$ compliance at fundamental

resonance frequency

$b_{\mathrm{P}} \quad$ velocity dependent friction in

$b_{\text {JDC }}$

primary path

velocity dependent friction in

secondary path

$c_{\mathrm{EF}} \quad$ spring stiffness of the equivalent frame

$c_{\text {JDC }} \quad$ spring stiffness of the jerk decoupling 


\begin{tabular}{|c|c|c|c|}
\hline$d_{\mathrm{EF}}$ & $\begin{array}{l}\text { specific damping of the equivalent } \\
\text { frame }\end{array}$ & $i_{\alpha, \beta}$ & $\begin{array}{l}\text { current in the st } \\
\text { coordinate syste }\end{array}$ \\
\hline$d_{\mathrm{JDC}}$ & $\begin{array}{l}\text { specific damping of the jerk } \\
\text { decoupling }\end{array}$ & $k_{\mathrm{P}, \mathrm{EF}}$ & $\begin{array}{l}\text { gain of the equi } \\
\text { parameter) }\end{array}$ \\
\hline$d w$ & differential work & $l_{\mathrm{s}}$ & inductance of li \\
\hline$D_{\mathrm{EF}}$ & $\begin{array}{l}\text { damping of the equivalent frame } \\
\text { (control parameter) }\end{array}$ & $\begin{array}{l}m_{\mathrm{EF}} \\
m_{\mathrm{JDC}}\end{array}$ & $\begin{array}{l}\text { mass of the equ } \\
\text { mass of the jerk }\end{array}$ \\
\hline$f_{\mathrm{c}}$ & parameter: forces founded by & $m_{\mathrm{P}}$ & mass of the prin \\
\hline & Coulomb [7] & $p$ & $\begin{array}{l}\text { pairs of poles } \\
\text { resistance of l }\end{array}$ \\
\hline $\begin{array}{l}f_{\mathrm{cEF}} \\
f_{\mathrm{cJDC}} \\
f_{\mathrm{d}}\end{array}$ & $\begin{array}{l}\text { spring force of the equivalent frame } \\
\text { spring force of the jerk decoupling } \\
\text { drive force }\end{array}$ & $\begin{array}{l}r_{\mathrm{s}} \\
t_{\mathrm{el}}\end{array}$ & $\begin{array}{l}\text { resistance of lin } \\
\text { electric delay ti } \\
\text { motor }\end{array}$ \\
\hline$f_{\mathrm{dEF}}$ & $\begin{array}{l}\text { damping force of the equivalent } \\
\text { frame }\end{array}$ & $u_{\alpha}, u_{\beta}$ & $\begin{array}{l}\text { voltages in the } \\
\text { coordinate syst }\end{array}$ \\
\hline$f_{\mathrm{dJDC}}$ & damping force of the jerk decoupling & $u_{d}$ & voltage in the \\
\hline$f_{\text {fric_c }}$ & forces founded by Coulomb [7] & $u_{u}, u_{v}, u_{w}$ & three phase vc \\
\hline$f_{\text {fric_s }}$ & forces founded by Stribeck $[\mathbf{8}]$ & $u_{q}$ & voltage in th \\
\hline$f_{\text {fric_v }}$ & forces founded by Reynolds [9] & $v$ & velocity in ge \\
\hline$f_{\text {fricjDC }}$ & friction force of the jerk decoupling & $v_{\text {act }}$ & actual speed \\
\hline$f_{\text {fricP }}$ & $\begin{array}{l}\text { friction force of the primary part from } \\
\text { the guides of a linear motor }\end{array}$ & $\begin{array}{l}x_{\mathrm{EF}} \\
x_{\mathrm{JDC}}\end{array}$ & $\begin{array}{l}\text { position of the } \\
\text { position of the } j\end{array}$ \\
\hline$f_{\mathrm{h}}$ & $\begin{array}{l}\text { parameter: forces founded by } \\
\text { Stribeck }[\mathbf{8}]\end{array}$ & $x_{\mathrm{P}}$ & $\begin{array}{l}\text { position of the } \\
\text { linear motor }\end{array}$ \\
\hline$f_{\mathrm{mEF}}$ & $\begin{array}{l}\text { acceleration force of the equivalent } \\
\text { frame }\end{array}$ & $\begin{array}{l}\theta_{\mathrm{r}} \\
\tau_{\mathrm{g}}\end{array}$ & $\begin{array}{l}\text { rotor angle in } \alpha \\
\text { pole width of lir }\end{array}$ \\
\hline$f_{\mathrm{mJDC}}$ & $\begin{array}{l}\text { acceleration force of the jerk } \\
\text { decoupling }\end{array}$ & $\begin{array}{l}\Delta E \\
\Phi\end{array}$ & $\begin{array}{l}\text { work, energy in } \\
\text { field flux linkag }\end{array}$ \\
\hline$f_{\mathrm{mP}}$ & $\begin{array}{l}\text { acceleration force of the primary part } \\
\text { from the linear motor }\end{array}$ & $\omega_{0, \mathrm{EF}}$ & $\begin{array}{l}\text { real angular res } \\
\text { the equivalent } \mathrm{f}\end{array}$ \\
\hline $\begin{array}{l}f_{\text {res1 }} \\
f_{\mathrm{v}}\end{array}$ & $\begin{array}{l}\text { fundamental resonance frequency } \\
\text { parameter: forces founded by } \\
\text { Reynolds }[\mathbf{9}]\end{array}$ & $\omega_{\mathrm{m} 1}$ & $\begin{array}{l}\text { angular resonar } \\
\text { equivalent fram }\end{array}$ \\
\hline$g_{\mathrm{EF}}$ & $\begin{array}{l}\text { transfer function of the equivalent } \\
\text { frame }\end{array}$ & & \\
\hline$g_{\text {JDC }}$ & $\begin{array}{l}\text { transfer function of the jerk- } \\
\text { decoupling slide }\end{array}$ & damp & damping \\
\hline$g_{\mathrm{P}}$ & transfer function of the primary part & $\mathrm{EF}$ & equivalent fram \\
\hline$g_{P}-J D C$ & transfer function of the linear motor, & elast & elastic \\
\hline & jerk-decoupling slides & $\begin{array}{l}\text { IrIC } \\
\text { JDC }\end{array}$ & jerk-decoupling \\
\hline$i_{d}$ & current in the direct axis & kin & kinetic \\
\hline$i_{q}$ & current in the quadrature axis & leak & leakage \\
\hline$i_{\mathrm{s}}$ & sum-current & $\mathrm{P}$ & primary \\
\hline$i_{u, v}$ & three-phase current at the motor & pot & potential \\
\hline
\end{tabular}

